\title{
BEMERKUNGEN ZUR BEWEISFÜHRUNG IN CICEROS CLUENTIANA
}

\section{Tamás Nótári*}

Die längste uns überlieferte und tatsächlich gehaltene oratio Ciceros ist die im Jahre 66, dh im Jahre seiner Prätur für Aulus Cluentius Habitus gehaltene Rede. Aus gewisser Hinsicht ist sie einer der Edelsteine der ciceronianischen Redekunst: Ihre Narrative ist lebhaft und spannend, wie die eines Kriminalromans, die Ereignisse, die Tatorte und die Zeitebenen wechseln sich in ihr gewagt und manchmal scheinbar unlogisch, ergeben aber zum Schluss eine präzis abgerundete, geschlossene, dem Ziel des Redners dienende Einheit. Gegen Cluentius, der dem Ritterstand (ordo equester) angehörte, wurde einerseits mit jener Begründung Anklage erhoben, dass er seinen Stiefvater, Statius Albius Oppianicus vergiftet haben soll. Andererseits wurde gegen ihn im Prozess auch vorgebracht, dass er vor acht Jahren in einem Prozess, den er gegen Oppianicus deswegen angestrengt hatte, weil dieser angeblich versucht hatte ihn zu ermorden, die Richter bestochen haben soll, was zur Folge hatte, dass Oppianicus den letzten Teil seines Lebens im Exil zu verbringen hatte. Den gesetzlichen Hintergrund der Anklage wegen Giftmordes bildete die lex Cornelia de sicariis et veneficiis aus dem Jahre 81, in der allerdings nur für jene Art der Richterbestechung eine Sanktion festgesetzt war, die von Mitgliedern des Senatorenstandes begangen wurde.

Zuerst sollte der historische Hintergrund der Rede und der gesetzliche Tatbestand, dh die Anwendbarkeit der lex Cornelia de sicariis et veneficiis untersucht werden.

* Wissenschaftlicher Hauptmitarbeiter, Rechtswissenschaftliches Institut des Gesellschaftswissenschaftlichen Zentrums der Ungarischen Akademie der Wissenschaften; Universitätsdozent, Károli Gáspár Universität Budapest.

\section{UNISA $\cong$}

Fundamina Volume 21 | Number 1 | 2015 pp 84-101
Doi: $10.17159 / 2411-7870 / 2015 / v 21 n 1 a 5$ Print ISSN 1021-545X/ Online ISSN 2411-7870 (c) Unisa Press 
Abschliessend sollen die von Cicero in der Cluentiana angewandten rhetorischen Mittel unter die Lupe genommen und aus jener Hinsicht behandelt werden, wie Cicero die Chronologie der Anklagepunkte in seiner auch juristisch brillanten Argumentation behandelt, verändert bzw entstellt hat.

1 Die Rede für Aulus Cluentius Habitus aus dem Jahre 66 wurde von Cicero in seinem zwanzig Jahre später geschriebenen Orator als Beispiel für die Verwendung der drei Stilarten in derselben Gerichtsrede erwähnt, ${ }^{1}$ und er zitiert daraus $^{2}$ diesbezüglich eine äusserst gelungene Formulierung. ${ }^{3}$ Als Quintilian über das Urteilsvermögen des Redners schreibt, beruft er sich auf die Cluentiana als Musterbeispiel der gut angelegten forensischen Strategie, ${ }^{4}$ anderswo stellt er wiederum fest, dass in der Cluentiana Cicero den Geschworenen Sand in die Augen gestreut hat. ${ }^{5}$ Die Rede für Cluentius wird von Gellius zitiert, ${ }^{6}$ Plinius der Jüngere hält sie für die hervorragendste rednerische Leistung Ciceros, ${ }^{7}$ von den Juristen beruft sich Claudius Tryphoninus auf sie. ${ }^{8}$ Theodor Mommsen würdigt die Cluentiana folgendermassen: „Die Criminalstatistik aller Zeiten und Länder wird schwerlich ein Seitenstück bieten zu einem Schaudergemälde so mannichfaltiger, so entsetzlicher und so widernatürlicher Verbrechen, wie es der Prozess des Aulus Cluentius in einem Schoss einer der angesehensten Familien einer italischen Ackerstadt vor uns aufgerollt." "9

Der Angeklagte des Prozesses, A Cluentius Habitus ist im nördlichen Apulien, in dem von den miteinander mehrfach verschwägerten Aurii, Albii, Cluentii und Magii dominierten Larinum geboren, ${ }^{10}$ das ein Spiegelbild der in Rom wuchernden Lastern zeigte $^{11}$ - und es kann nicht einmal behauptet werden, dass der Grösse der Landstadt entsprechend. ${ }^{12}$ Seinen Vater, Cluentius den Älteren, verlor er im Jahre 88, als er fünfzehn Jahre alt war, ${ }^{13}$ seine Mutter, Sassia heiratete zwei Jahre später ihren

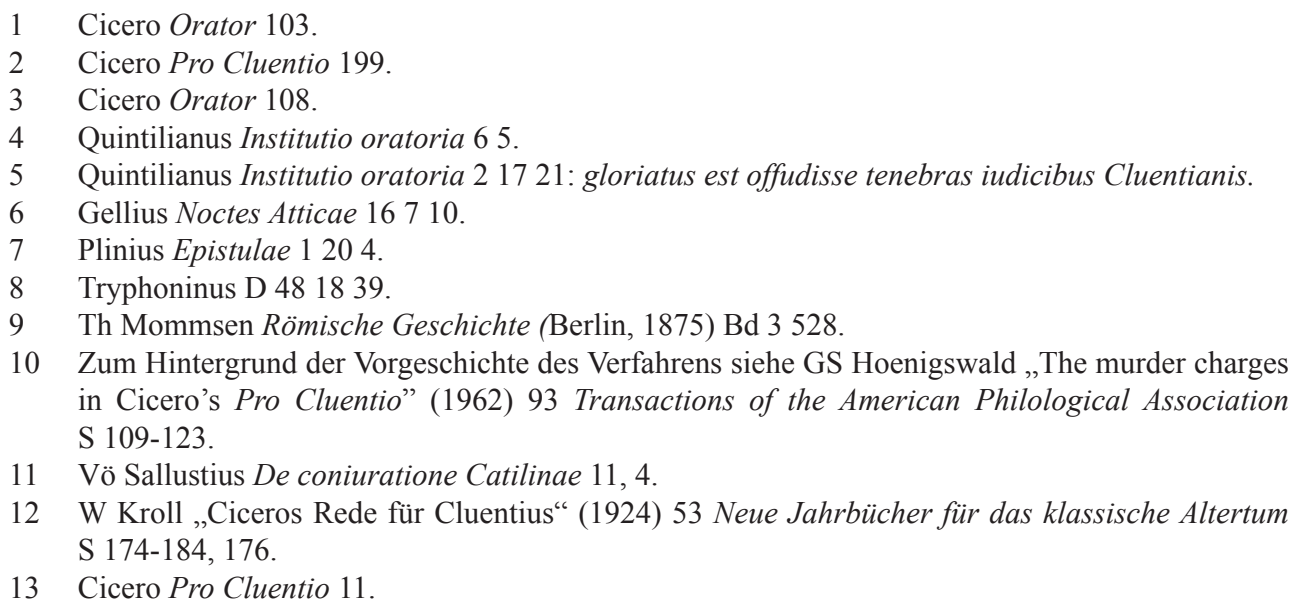

0 Zum Hintergrund der Vorgeschichte des Verfahrens siehe GS Hoenigswald „The murder charges in Cicero's Pro Cluentio" (1962) 93 Transactions of the American Philological Association S 109-123.

11 Vö Sallustius De coniuratione Catilinae 11, 4.

12 W Kroll „Ciceros Rede für Cluentius“ (1924) 53 Neue Jahrbücher für das klassische Altertum S 174-184, 176.

13 Cicero Pro Cluentio 11. 
Schwiegersohn A Aurius Melinus, den geschiedenen Gatten ihrer Tochter Cluentia. ${ }^{14}$ Cicero datiert von hier an das schlechte Verhältnis zwischen dem Angeklagten und seiner Mutter und behauptet, dass ihn Sassias Verhalten dermassen empört hatte, dass er beschloss, mit seiner Mutter keinerlei Kontakt pflegen zu wollen. ${ }^{15}$ Aurius fiel angeblich in Folge der Machenschaften des St Abbius Oppianicus - den sullanischen Proskriptionen zum Opfer. ${ }^{16}$ Sassia heiratete Oppianicus, der sich schon von zwei Ehefrauen, von Papia (der Witwe des Magius) und Novia getrennt hatte bzw zwei Gattinnen, Cluentia die Ältere und Magia verloren hatte. ${ }^{17}$

Es ist erwähnenswert, dass Cicero, als er vom Hass zwischen Oppianicus dem Älteren und Cluentius spricht, von jenem Element Gebrauch macht, das er als psychologische Motivation des von Oppianicus auf seinen Stiefsohn verübten Giftmordversuches hätte verwenden können, nämlich dass Cluentius auf die Heirat zwischen Sassia und Oppianicus mit Antipathie und Groll reagiert hat. ${ }^{18}$ Oppianicus der Jüngere, der im Jahre 66 gegen Cluentius als Ankläger auftrat, stammte von Magia, einer früheren Gattin des Oppianicus des Älteren. Oppianicus der Ältere wollte angeblich seinen Stiefsohn Cluentius vergiften lassen: Zur Ausführung seines Plans nahm er die Hilfe des C Fabricius in Anspruch, der zusammen mit dem Freigelassenen Scamander, den Sklaven des Cluentius behandelnden Arztes, für sein Vorhaben gewinnen wollte. ${ }^{19}$ In wie fern der Mordversuch als bewiesen gelten kann, mag dahingestellt bleiben, Tatsache ist, dass Cluentius zuerst gegen Scamander und Fabricius bzw abschliessend gegen seinen Stiefvater, Oppianicus den Älteren Anklage erhob. Oppianicus wurde vom Gericht mit einer ganz knappen Stimmenmehrheit für schuldig befunden und verurteilt. ${ }^{20}$ Den Prozess umgaben mehrere verdächtige Umstände, so zB wurden die Richter nicht vorschriftsgemäss ausgelost $\mathrm{t}^{21}$ und der Bestechungsverdacht ${ }^{22}$ betraf mehrere Senatoren, unter anderen C Fidiculanius Falcula, ${ }^{23} \mathrm{M}$ Atilius Bulbus und Staienus. ${ }^{24}$

Aufgrund all dessen schlug der Verdacht Wurzel, dass das Urteil im Prozess gegen Oppianicus den Älteren von bestochenen Richtern gefällt wurde. Cicero versucht - trotz der Verurteilung des Oppianicus - die Lage so darzustellen, dass die Bestechung von Oppianicus selber vorgenommen wurde, und er nur diesem zu verdanken hatte, dass beinahe die Hälfte der Richter für seinen Freispruch

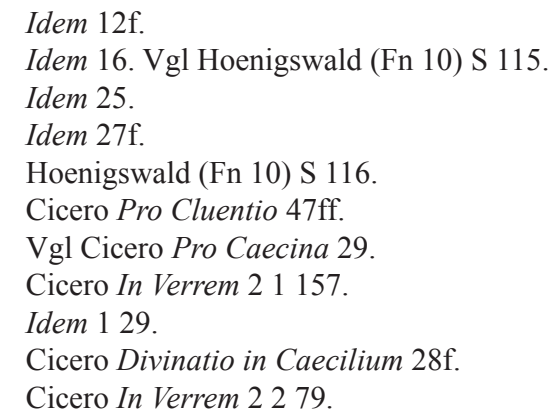


stimmten, im Gegensatz zur einstimmigen Verurteilung des Scamander und des Fabricius. L Quinctius, der Verteidiger des Oppianicus verdächtigte Cluentius der Richterbestechung, da seiner Anklage im Endeffekt Erfolg beschieden war, und gebrauchte als Volkstribun gerade diesen Fall als Beispiel vor der Volksversammlung, um gegen die senatorischen Gerichtshöfe zu agieren. ${ }^{25}$ Der Prozess schlug politische Wellen und mehrere Senatoren, die am Verfahren als Richter beteiligt waren, wurden wegen Korruption verurteilt. ${ }^{26}$ Cicero, der im Verfahren im Jahre 74 als Verteidiger Scamanders auftrat, erwähnte den Prozess gegen Oppianicus gerade wegen der Verurteilung des Angeklagten mit einer knappen Stimmenmehrheit, woraus er Beweise, oder zumindest Indizien auf die Bestechung der Richter durch Oppianicus, als Paradebeispiel der Korruptheit des Gerichtswesens bringen wollte. ${ }^{27}$

Zwei Jahre nach seiner Verurteilung, dh im Jahre 72, starb Oppianicus der Ältere in der Nähe von Rom; ${ }^{28}$ der Ankläger behauptete, dass ihn Cluentius vergiften liess, ${ }^{29}$ aber von den Umständen seines Todes ist uns nichts Genaueres bekannt. Seine Witwe, Sassia, verdächtigte ihren Sohn (dh den Stiefsohn des Oppianicus) damit, dass er Oppianicus vergiftet haben soll, und versuchte ihren Verdacht durch Beweise, in erster Linie durch Geständnisse ihrer Sklaven, die sie einer Tortur unterziehen liess, zu untermauern - allerdings mit wenig Erfolg. ${ }^{30}$ Nachdem Cluentius in mehrere Todesfälle, die sich in der Zwischenzeit ereignet haben, verwickelt worden war, erhob im Jahre 66 aufgrund der lex Cornelia de sicariis et veneficiis der junge - zum Zeitpunkt des Prozesses ungefähr einundzwanzigjährige ${ }^{31}$ - Abbius Oppianicus gegen Cluentius Anklage. Das Mordgesetz Sullas umfasste mehrere Tatbestände: Todschlag, unerlaubtes Tragen von Waffen, Herstellung und Verkauf bzw Verabreichung von Gift in Tötungsabsicht, Brandstiftung und einige Straftaten im Zusammenhang mit dem Kriminalverfahren, so zB die Bestechung von Richtern, um mit deren Beihilfe Unschuldige verurteilen zu lassen - das letztere Tatbestandselement bezog sich allerdings nur auf Magistrate und Senatoren. ${ }^{32}$

25 Cicero Pro Cluentio 74ff.

26 CJ Classen Recht, Rhetorik und Politik, Untersuchungen zu Ciceros rhetorischer Strategie (Darmstadt, 1985) S 21.

27 Cicero In Verrem $138-40$.

28 Kroll (Fn 12) S 174.

29 Vö Cicero Pro Cluentio $161 \mathrm{ff}$.

30 Hoenigswald (Fn 10) S 111; Kroll (Fn 12) S 175.

31 W Stroh Taxis und Taktik, Die advokatische Dispositionskunst in Ciceros Gerichtsreden (Stuttgart, 1975) S 195.

32 Vgl Th Mommsen Römisches Strafrecht (Leipzig, 1899) S 628; W Kunkel Untersuchungen zur Entwicklung des römischen Kriminalverfahrens in vorsullanischer Zeit (München, 1962) S 6470; JD Cloud „The primary purpose of the lex Cornelia de sicariis“ (1969) 86 Zeitschrift der Savigny-Stiftung für Rechtsgeschichte, Romanistische Abteilung S 258-268; CJ Classen „Cicero, Pro Cluentio 1-11 im Licht der rhetorischen Theorie und Praxis“ (1965) 108 Rheinisches Museum S 104-142, 140; J Humbert „Comment Cicéron mystifia les juges de Cluentius“ (1938) 16 Latomus, Revue des Etudes Latines S 275-296, 276. 
Cluentius gehörte - wie gesagt - dem Ritterstand an, und hatte kein Amt innegehabt, so hätte er nach dem Wortlaut des Gesetzes keine Bestechung ausüben können, die unter das besagte Gesetz fiel..$^{33}$ Das Amt des iudex quaestionis versah Q Voconius Naso, ${ }^{34}$ als Nebenankläger trat neben Oppianicus Titus Attius, ein junger Ritter aus Pisaurum auf. ${ }^{35}$ Die Verteidigung des - in jenen Anklagepunkten, die gegen ihn vorgebracht wurden, mit grösster Wahrscheinlichkeit schuldigen - Cluentius übernahm der im Jahre des Prozesses als Prätor fungierende Cicero, der mit seiner rednerischen Brillanz den Freispruch seines Klienten erwirken konnte. ${ }^{36}$ Den Gerichtshof bildeten zweiunddreissig Richter, die nach der im Jahre 70 erbrachten lex Aurelia iudiciaria zu je einem Drittel aus dem Senatorenstand, aus dem Ritterstand und dem Stand der Ärartribunen hervorgingen. ${ }^{37}$

Die ciceronianische Verteidigung läuft auf zwei Linien, der Redner wendet sich nicht sofort der Hauptanklage zu, sondern verweilt länger bei der Frage der Richterbestechung. Um seine eigene Theorie von der Richterbestechung zu belegen, behandelt er das Thema der Bestechung wahrscheinlich viel ausführlicher, als der Ankläger darauf eingegangen ist: Er schildert detailliert das üble Vorleben des Oppianicus und widmet jenen zwei Prozessen, die mit dem versuchten Attentat gegen Cluentius in Verbindung stehen, grosse Aufmerksamkeit. In seiner Einleitung kündet Cicero an, dass er der von dem Ankläger angelegten Thematik folgen will, bzw er gibt darüber Rechenschaft, warum er sich viel ausführlicher mit dem ersten, als mit dem zweiten Anklagepunkt auseinander zu setzen beabsichtigt. Hiernach sollte der Anklagepunkt des Giftmordes vollkommen unbegründet sein und könnte mit wenigen Worten abgetan werden, jener der Richterbestechung hätte sich aber in den letzten acht Jahren dermassen im Bewusstsein aller festgesetzt, dass es der einträchtigen Anstrengung der Richter und des Verteidigers bedurfte, diese Nachrede aus der öffentlichen Meinung auszumerzen. Der erste Teil der Rede besteht aus drei grösseren Kapiteln, die sich mit dem Vorleben des Oppianicus des Älteren, mit dem Giftmordprozess aus 74 und mit dem Tatbestand der Richterbestechung befassen. Im zweiten Teil der Cluentiana, der nun von dem eigentlichen Anklagepunkt, dh von der Ermordung des Oppianicus durch Cluentius handelt, werden die dunklen Kapitel des Vorlebens des Cluentius und auch die Ermordung des Oppianicus von Cicero in lapidarer Kürze und mit verdächtiger Leichtigkeit abgetan: Eine verhältnismässig längere Ausführung erfährt nur das vom Ankläger als Beweis vorgebrachte Geständnis, das den Sklaven durch Folter entnommen wurde.

33 Stroh (Fn 31) S 196.

34 Cicero Pro Cluentio $147 \mathrm{f}$.

35 Idem 6584 156; Brutus 271.

36 Kroll (Fn 12) S 174.

37 Stroh (Fn 31) S 202. 
2 Gleich im prooemium trennt Cicero die Anklage wegen Giftmordes von jener, die von dem Nebenankläger Attius vorgebracht wurde und sich auf die angebliche Bestechung jenes Gerichtshofes bezog, die vor acht Jahren Oppianicus verurteilt hatte. ${ }^{38}$ Die genaue Bestimmung der Anklagepunkte, die dem Prozess gegen Cluentius zugrunde gelegen haben müssen, scheint keines Wegs einfach zu sein: Die Anklage hätte sich erstens auf Meuchelmord und Giftmischerei, zweitens auf Giftmordversuch und Bestechung des Gerichts, drittens auf Mordversuch richten können. ${ }^{39}$ Die Rekonstruktion der Fakten wird auch noch dadurch erschwert, dass Cicero einerseits manche Umstände, die schlechtes Licht auf seinen Klienten werfen könnten wissentlich verschweigt, und andererseits einige, für den Zeitgenossen selbstverständliche, für den heutigen Leser aber nicht hinreichend bekannte Elemente übergeht. Ciceros Aufgabe war es, da er die Verteidigung des Cluentius übernahm, den Richtern überzeugend darzulegen, dass sein Klient jene Straftat(en), deren er angeklagt worden war, nicht begangen haben kann, dh er hatte gemäss der rhetorischen Theorie nach der zu dem status coniecturalis passenden Argumentationsweise vorzugehen.

Aus rechtshistorischer Hinsicht ist eine der interessantesten Fragen, die sich in Verbindung mit der Cluentiana ergeben, ob sich jene Anklage, die vom jüngeren Oppianicus gegen Cluentius aufgrund der lex Cornelia de sicariis et veneficiis erhoben wurde, nur auf den Giftmord oder aber auch auf die Bestechung der Richter bezog, die Cluentius vor acht Jahren im Prozess gegen Oppianicus den Älteren begangen haben soll. Bei der Klärung dieses Problems stellt sich natürlich die Frage nach der Zuverlässigkeit der Quellenbasis: Die ciceronianische Darstellungsweise und seine Verweise auf den Gesetzestext sind mit grosser Wahrscheinlichkeit tendenziös - selbst wenn er bei der Zitierung des Gesetzes keine wesentlichen Änderungen hat vornehmen können -, und die uns überlieferte Form der sullanischen Gesetze entstammt einer späteren Epoche ${ }^{40}$ was wiederum vermuten lässt, dass der zum Zeitpunkt des Prozesses geltende Gesetzestext mit dem uns bekannten nicht unbedingt als identisch gelten dürfte. ${ }^{41}$ Die spätere Fassung der lex Cornelia de falsis sanktioniert zwar die aktive Form der Bestechung des Gerichts, es erscheint allerdings als unwahrscheinlich, dass die ursprüngliche lex Cornelia testamentaria ebenfalls eine solche Bestimmung enthalten hätte. Beim

38 Cicero Pro Cluentio 1-2 11 119. Vgl Humbert (Fn 32) S 287; CJ Classen „Cicero, the Laws, and the Law-Courts“ (1978) 37 Latomus, Revue des Etudes Latines S 597-619, 604ff.

39 CJ Classen „Die Anklage gegen A. Cluentius Habitus (66 v. Chr. Geb.)“ (1972) 89 Zeitschrift der Savigny-Stiftung für Rechtsgeschichte, Romanistische Abteilung S 1-17; Ch Köhler Die Prö̈mientechnik in Ciceros Reden: Ein Beitrag zum Verhältnis von rhetorischer Theorie und rednerischer Praxis bei Cicero. Dissertation (Jena, 1968) S 100-109; G Pugliese „Aspetti giuridici della Pro Cluentio di Cicerone“(1970) 21 Iura S 155-181.

40 Paulus Sententiae 5 23; Collatio legum Mosaicarum et Romanarum 12 3; D 48 8. Vgl Mommsen (Fn 32) S 628ff; Cloud (Fn 32) S 258ff.

41 Classen (Fn 39) S 2. 
Bestimmungsversuch der Anklagepunkte darf auch nicht ausser Acht gelassen werden, dass im Quästionsprozess der Ankläger den Richtern alles vortragen durfte, was er gegen den Angeklagten vorbringen konnte, da sein Ziel nur ein allgemeiner Schuldspruch, und nicht die Feststellung der Schuld in den einzelnen Punkten war bzw die Festlegung der Strafe nicht dem Ermessen des Gerichtshofes oblag. ${ }^{42}$ Es ist nicht mit Sicherheit zu bestimmen, ob bei der delatio nominis neben dem Gesetz, das der Anklage zugrunde gelegt werden sollte, auch die genauen Passagen und die anderen, im Kreise der Anklage vorzubringen gedachten Punkte genannt werden sollten bzw ob diese im weiteren Laufe des Prozesses von zwingender Kraft waren. ${ }^{43}$

Es ist mit gutem Grund anzunehmen, dass bei der Einleitung des Prozesses die Anklage schriftlich fixiert werden musste, was auch in Bezug auf die vorsullanische Periode von Cicero in seinem Werk De inventione festgehalten wird. ${ }^{44}$ Es lohnt sich, etwas genauer unter die Lupe zu nehmen, in welchem Mass Cicero in seinen Reden die gesetzliche Grundlage des Verfahrens präzisiert bzw auf die Prozesseinleitung (postulatio, delatio nominis, receptio nominis) reflektiert. In seinen Gerichtsreden lassen sich direkte Hinweise auf den Tatbestand des crimen repetundarum, ${ }^{45}$ des crimen maiestatis,${ }^{46}$ des ambitus,${ }^{47}$ des peculatus, ${ }^{48}$ des crimen inter sicarios und des crimen veneficii, ${ }^{49}$ der iniuria,${ }^{50}$ des furtum, ${ }^{51}$ der vis,${ }^{52}$ der alea ${ }^{53}$ und des crimen parricidii ${ }^{54}$ finden. ${ }^{55}$ In zahlreichen Fällen beruft er sich auf ein konkretes Gesetz, so $\mathrm{zB}$ auf die lex Plautia de vi, ${ }^{56}$ die lex Iulia de pecuniis repetundis,${ }^{57}$ die lex Papia ${ }^{58}$ die lex Acilia ${ }^{59}$ und die lex Scantinia ${ }^{60}$ In einigen seiner Reden verweist er direkt auf die gesetzliche Grundlage der Anklagepunkte, so unter anderen in der Rede für

Idem S 3 .

Mommsen (Fn 32) S $385^{4}$.

Cicero De inventione 258.

45 Cicero Divinatio in Caecilium 76; In Verrem 22 142; ad Quintum fratrem 31 15; ad familiares 8 823.

46 Cicero ad Quintum fratrem 31 15; De inventione 2 72; ad familiares 32 3; Philippicae 123.

47 Cicero Pro Caelio 16 76; De oratore 2274 280; ad Quintum fratrem 12 15; 23 5; 32 3; Pro Cluentio 114.

48 Auctor ad Herennium 122.

49 Cicero De inventione 2 58; Pro Roscio Amerino 90; Pro Cluentio 21; Auctor ad Herennium 4, 23.

50 Cicero De domo sua 13; De inventione 2, 59.

51 Cicero Pro Cluentio 163; ad familiares 7, 22; Pro Flacco 43.

52 Cicero post reditum in senatu 19; ad Quintum fratrem 2, 3, 5; Pro Sestio 9095.

53 Cicero Philippicae 2, 56.

54 Cicero Pro Roscio Amerino 2864.

55 Classen (Fn 39) S 5.

56 Cicero ad familiares 881.

57 Cicero Pro Rabirio Postumo 12.

58 Cicero Pro Balbo 52.

59 Cicero In Verrem 2126.

60 Cicero ad familiares 812 3; 8144. 
Sextus Roscius aus Ameria, ${ }^{61}$ in der gegen Verres, ${ }^{62}$ in der für Scaurus,${ }^{63}$ in der für Rabirius Postumus ${ }^{64}$ und in der für Ligarius. ${ }^{65}$

In der Cluentiana bedient sich Cicero einer janusartigen Darstellungsweise. Einerseits erweckt er den Eindruck, als ob das Gerichtshof ausschliesslich für den Giftmord zuständig sei, ${ }^{66}$ da jene Passage der lex Cornelia de sicariis et veneficiis, die sich mit der Bestechung des Gerichtes durch die Mitglieder des Ritterstandes auseinandersetzt, sich auf Cluentius, der zum ordo equester gehört, nicht bezieht; ${ }^{67}$ andererseits bringt er den Anklagepunkt der Bestechung immer wieder zur Sprache. Die unter dem Vorsitz des Q Voconius Naso tagende quaestio war zweifelsohne in erster Linie für die Giftmorde zuständig - was natürlich nicht ausschloss, dass andere Anklagepunkte nicht hätten vorgebracht werden können -, aber ihre Zuständigkeit konnte auch auf die erwähnten Bestechungsdelikte ausgedehnt werden. ${ }^{68}$ Es lohnt sich allerdings, etwas länger bei den von Joachim Classen erwähnten Anklagepunkten zu verweilen, um den in der Anklage erfassten Tatbestand genauer definieren zu können. Trotz der lückenhaften Quellenbasis kann festgestellt werden, dass in anderen Fällen aufgrund der lex Cornelia de sicariis et veneficiis keine Anklagen wegen Richterbestechung erhoben wurden, da sich auch andere Möglichkeiten zur Verfolgung dieser Straftat boten. Es ist ausserdem höchst unwahrscheinlich, dass der iudex quaestionis eine Anklage angenommen hätte, die dem Wortlaut des Gesetzes zuwiderläuft, oder - mit anderen Worten - zugelassen hätte, dass der Ankläger den Tatbestand des sullanischen Gesetzes mit einer interpretatio extensiva auch auf den Ritterstand ausdehnt. Nach Cicero soll sich Attius mehrere Male auf die aequitas berufen und für die extensive Interpretation des Gesetzes plädiert haben. Im Gegensatz zu ihm behandelte Cicero - obwohl er bestrebt war Cluentius von den Folgen des Verdachtes der Richterbestechung zu verschonen - die vor acht Jahren angeblich vorgefallene Bestechung des Gerichtshofes an keinem Punkt als ein wirkliches crimen, bzw. er zitierte keine einzige Zeugenaussage, die dies widerlegen sollte, sondern tat dieses Element als ein vom Ankläger vorgebrachtes Gerücht ab, ${ }^{69}$ das die Gefahr in sich barg, bei den Richtern Antipathie und Voreingenommenheit gegen Cluentius zu generieren. ${ }^{70}$

61 Cicero Pro Roscio Amerino 28616476.

62 Cicero In Verrem 22141.

63 Cicero Pro Scauro 1.

64 Cicero Pro Rabirio Postumo 8937.

65 Cicero Pro Ligario 145911.

66 Cicero Pro Cluentio 12148164.

67 Idem 144ff.

68 Classen (Fn 39) S 10f.

69 Vgl Cicero Pro Cluentio 142.

70 Classen (Fn 39) S 14f. 
3 Die Behandlung der crimina veneficii, dh der tatsächlichen, juristisch relevanten Anklagepunkte an erster Stelle konnte in den Richtern jenen Eindruck erwecken, als ob Cicero versuchte, das iudicium Iunianum, den juristisch eher irrelevanten, jedoch höchst effektvollen Teil der Anklage zu umgehen und sich allerdings nur scheinbar, da er nur später auf den Oppianicusprozess zu sprechen kommt ${ }^{71}$ - dem von Attius erstellten System zu fügen. Wegen der Zwangswahl zwischen dem status collectionis und dem status coniecturalis entschloss sich Cicero zu einem halsbrecherischen, jedoch bereits in der Rosciana mit Erfolg angewandten ${ }^{72}$ Versuch: Er trennte seine eigenen Interessen bzw Forderungen von denen seines Klienten, indem er behauptete, dass er sich als Verteidiger nur auf den Wortlaut des Gesetzes hätte berufen müssen, ${ }^{73}$ sich aber dennoch - um den Wunsch des Cluentius, der nicht nur seinen Freispruch erreichen, sondern auch seinen guten Ruf wiederherstellen lassen wollte, nachzukommen ${ }^{74}$ - für den schwierigeren Weg entschieden hatte, der darin bestand, die Unschuld des Angeklagten nicht nur formell, sondern auch materiell zu beweisen. ${ }^{75}$ Hiermit gelang es ihm, den Eindruck zu erwecken, als ob jeder einzelne der beiden status an und für sich genügend Gewicht besessen hätte, den Sieg im Prozess zu sichern. ${ }^{76}$

Die von Cicero angewandte, auf zwei Spuren laufende Argumentation war auch den Erwartungen der Geschworenen angepasst. Einerseits verteidigte er mit der Anwendung des status collectionis die Interessen des Ritterstandes, indem er sich an den Wortlaut des Gesetzes hielt, der für sie die Straffreiheit vorsah, ${ }^{77}$ und erweckte in ihnen zugleich jene Angst, dass im Falle einer extensiven Interpretation in der Zukunft auch gegen Ritter Anklage wegen Richterbestechung erhoben werden könnte. ${ }^{78}$ Andererseits brauchte sich Cicero nicht davor zu fürchten, sich den Zorn der Richter aus dem Senatorenstand zuzuziehen, da er sich mit der Anwendung des status coniecturalis ihre Sympathie gesichert hatte, indem er beweisen konnte, dass sich im iudicium Iunianum Oppianicus, und nicht Cluentius der Richterbestechung schuldig gemacht hatte bzw. dass nur einige Richter bestochen worden waren und Staienus als einziger Geld erhalten hatte, ${ }^{79}$ wodurch es ihm gelang, die durch die Ereignisse des Jahres 74 angeschlagene Ehre des Richterstandes wiederherzustellen. ${ }^{80}$ Die Disposition der Cluentiana lässt sich - wie Stroh zutreffend festgestellt hat ${ }^{81}$

71 Cicero Pro Cluentio 59ff.

72 Cicero Pro Roscio Amerino 128ff.

73 Cicero Pro Cluentio 145.

74 Idem 144.

75 Stroh (Fn 31) S 200.

76 Quintilianus Institutio oratoria 659.

77 Cicero Pro Cluentio 150-155.

78 Idem 152 157. Zur Gerichtsbestechungs Mommsen (Fn 32) S $634 f$.

79 Kroll (Fn 12) S 178.

80 Stroh (Fn 31) S 203.

81 Idem S 204. 
- folgendermassen aufteilen: In der Erörterung des iudicium Iunianum ${ }^{82}$ werden durch den status coniecturalis (dh mit jener Behauptung, dass Cluentius den Giftmord nicht verübt hatte) die Senatoren ${ }^{83}$ durch den status collectionis (dh mit jener Argumentation, dass aufgrund des sechsten Paragraphen der lex Cornelia de sicariis et veneficiis Cluentius nicht strafbar sei) die Ritter angesprochen ${ }^{84}$ worauf die Behandlung der crimina veneficii folgt. ${ }^{85}$

Um der dem jüngeren Oppianicus zuströmenden Sympathie jegliche Bedeutung zu nehmen, greift Cicero zu einem meisterhaften Mittel: Er lässt die Mutter des Cluentius (dh die Witwe des Oppianicus des Älteren bzw die Stiefmutter des Oppianicus des Jüngeren), die gegenüber ihrem eigenen Sohn von hostile odium und crudelitas erfüllte Sassia, ins Bild treten, in deren Hand - da angeblich sie diejenige sein sollte, die die Fäden der Anklage zog - selbst der von pietas geleitete jüngere Oppianicus zum Rachewerkzeug wird. ${ }^{86}$ Es lohnt sich, genauer zu untersuchen, an welchen Punkten und in welchem Kontext Sassia von Cicero erwähnt wird. ${ }^{87}$

Unmittelbar nach dem Exordium nennt er Sassia, die von Grausamkeit und Hass geleitete Mutter, als Quelle der Anklage. ${ }^{88}$ Jene Frage, ob Sassia (wie Classen behauptet) bei der Verhandlung anwesend war, ${ }^{89}$ oder (wie Stroh und Humbert vermuten) nicht, ${ }^{90}$ ist - da sie weder von Cicero angesprochen wird, noch wir davon unterrichtet sind, dass sie in den Zeugenstand getreten wäre - nicht zu entscheiden und vielleicht auch nicht von ausschlaggebender Relevanz. Schon hier betont er mit Nachdruck, dass Sassia eine überaus bedeutende Rolle im Prozess spielt ${ }^{91}$ und dass er sie - im Interesse des Cluentius - nicht schonen kann, ${ }^{92}$ aber worin all dies genau besteht, erfahren wir erst später, nämlich bei der Behandlung der crimina veneficii. ${ }^{93}$ Das Protokoll vom Sklavenverhör liess Attius vor Gericht vorlesen,${ }^{94}$ es ist jedoch fraglich, ob der Name der Sassia darin vorkam. ${ }^{95}$ Diese - mit grosser Wahrscheinlichkeit höchst subjektive und an rhetorischen Übertreibungen reiche Darstellung der Ereignisse, die wohl kaum als Rekonstruktion genannt werden kann, bot Cicero eine willkommene Gelegenheit, eine effektvolle Invektive gegen Sassia

82 Cicero Pro Cluentio 9-160.

83 Idem 9-142.

84 Idem 143-160.

85 Idem 161-187.

86 Idem 12ff. Vgl Quintilianus Institutio oratoria 65 9; 11162.

87 Stroh (Fn 31) S 205ff.

88 Cicero Pro Cluentio $12 \mathrm{ff}$.

89 Classen (Fn 26) S 36.

90 Stroh (Fn 31) S 206; J Humbert Les plaidoyers écrits et les plaidoiries réelles de Cicéron (Paris, 1925) S $115 \mathrm{ff}$.

91 Cicero Pro Cluentio 17.

92 Idem 18.

93 Idem $176 \mathrm{ff}$.

94 Idem 184.

95 Stroh (Fn 31) S 206. 
zu beginnen. ${ }^{96}$ Jenes frühere Versprechen von einer detaillierten Ausführung ${ }^{97}$ löst der Redner nur nach dieser Invektive ein und führt den vom Verhältnis zwischen dem Angeklagten und dessen Mutter vor der Verhandlung mit grosser Wahrscheinlichkeit nichts ahnenden Richtern das Bild der zum Monster stilisierten unmütterlichen Mutter vor Augen. Nach dieser Darstellung war sie schon von Anfang an am Mordversuch gegen Cluentius beteiligt, ${ }^{98}$ er machte ihren Stiefsohn zu ihrem Schwiegersohn, um in seiner Person einen geeigneten Ankläger gegen ihren leiblichen Sohn stellen zu können. ${ }^{99}$ Nach der kurzen Zusammenfassung des Sklavenverhörs ${ }^{100}$ schafft der Redner das Bild der Zeugen manipulierenden, das Verderben ihres Sohnes herbeiführenden, nach Rom geeilten, die Fäden der Anklage in ihrer Hand haltenden und sich vor der Öffentlichkeit im Verborgenen haltenden Sassia. ${ }^{101}$

Da die Ankläger Sassia wahrscheinlich unerwähnt liessen und den Gegensatz vom pius Oppianicus und vom impius Cluentius den Richtern einzuprägen versuchten, liess Cicero - indem er das Mittel der retorsio criminis in Anspruch nahm - mit gutem Gefühl die von den Anklägern erstellte Charakterisierung auf sie zurückfallen: Den jüngeren Oppianicus, der wegen seines jugendlichen Alters bei den Richtern einen guten Eindruck hinterliess, konnte Cicero nicht angreifen, anstatt seiner wählte er Sassia zu seiner Zielscheibe, die zu diesem Zweck umso geeigneter zu sein schien, weil ihre um 86 mit ihrem Schwiegersohn eingegangene Ehe ${ }^{102}$ in den Richtern den Topos eines die Gesetze der Natur missachtenden und auch vor Verbrechen nicht zurückschreckenden Weibes bestärkte. ${ }^{103}$ Sein Ziel erreicht Cicero mit der genialen Umstrukturierung der Ereignisse, denn ihm wird von dem ordo artificiosus ermöglicht, die zweigeteilte narratio in die argumentatio einzubauen und von hieraus auf geradem Wege zur peroratio, die zugleich die Rolle einer Invektive gegen Sassia einnimmt, zu gelangen. Hiernach soll sich die Aufmerksamkeit und das Bestreben der Richter nicht mehr darauf richten, den Fall des für die Verurteilung und den Tod seines Stiefvaters auf Rache sinnenden jüngeren Oppianicus zu erwägen, sondern darauf, Cluentius von der Rache seiner Mutter zu retten, die alle Gesetze des menschlichen Zusammenlebens mit Füssen tritt, und um ihr Ziel zu erreichen, die Justiz hierzu ats missbrauchen will. ${ }^{104}$

In dem Teil der Rede, in dem er das iudicium Iunianum behandelt, ${ }^{105}$ vermischt Cicero die traditionell gut trennbaren Mittel der narratio und der argumentatio

96 Cicero Pro Cluentio 176-187.

97 Idem 17.

98 Idem 189.

99 Idem 190. Vgl Kroll (Fn 12) S 175; Hoenigswald (Fn 10) S 111.

100 Cicero Pro Cluentio 191.

101 Idem 192ff.

102 Idem 12.

103 Hoenigswald (Fn 10) S 113.

104 Stroh (Fn 31) S 210.

105 Cicero Pro Cluentio 9-142. 
mit grossem Geschick. Nach der propositio ${ }^{106}$ und der dazwischen geflochtenen Erzählung über Sassia ${ }^{107}$ beginnt er eine in der confirmatio gipfelnden ${ }^{108}$ narratio, aber deren gewisse Teile, ${ }^{109}$ so zB die Greueltaten des Oppianicus ${ }^{110}$ und die praeiudicia erzählenden Paragraphen ${ }^{111}$ erfüllen den Zweck des probabile e causa ${ }^{112}$ da sie jene Behauptung zu unterstreichen berufen waren, dass es nicht Cluentius, sondern Oppianicus im Interesse gestanden hat, das Gericht zu bestechen. ${ }^{113}$ Genauso geht die Argumentation über das Bestechungsgeld als probabile e facto dem narrativen Teil einerseits voraus, ${ }^{114}$ andererseits folgt sie ihm, ${ }^{115} \mathrm{dh}$ sie umschliesst die hiervon handelnde narratio. ${ }^{116}$ Jene Argumentation also, in der sich die ungefähre Chronologie der Ereignisse abzeichnet, trägt einen narrativen Charakter. ${ }^{117}$

Dieses komplizierte Vorgehen ist unerlässlich, damit Cicero den Richtern jenen (ziemlich unwahrscheinlichen) Gedankengang akzeptabel machen kann, dass im Prozess des Jahres 74 nicht der obsiegende Cluentius, sondern der für schuldig erklärte Oppianicus den Gerichtshof bestochen hat, und zwar auf jene Weise, dass der von ihm gedungene Mittelsmann, Staienus, den Richtern das Bestechungsgeld zwar versprochen, später aber behauptet hatte, dass der Angeklagte nicht bereit sei, zu zahlen, um die Richter gegen ihn aufzuwiegeln und sichern zu können, dass Oppianicus verurteilt werde und er die ganze Summe behalten könnte. Cicero konnte aber seinen Hörern diese Erzählung nicht ohne Vorbereitung auftischen, so war er gezwungen, die rekonstruktive narratio mit der vorausgehenden argumentatio zu untermauern und wahrscheinlich zu machen, dass Oppianicus - in Anbetracht seiner zahlreichen Untaten und jener praeiudicia, die seinen Fall negativ beeinflussten - gewichtige Gründe gehabt haben muss, das Gericht zu bestechen. ${ }^{118}$ Cicero gerät hier in Widerspruch mit jenem Versprechen, dass er in seiner Rede die vom Gegner aufgestellte Gliederung befolgen will, ${ }^{119}$ obwohl er sein Versprechen in der eigentlichen narratio mehr oder minder einlöst, trotz jenes Umstandes, dass er zuvor auf mehrere, vom Ankläger nicht berührte Punkte zu sprechen kommt. In der langen Einleitung versichert Cicero die Richter dessen, dass er sich nunmehr kurz fassen

106 Idem 9-11.

107 Idem 11-18.

108 Idem 81.

109 Idem 21-61.

110 Idem $21 \mathrm{ff}$.

111 Idem 49ff.

112 Stroh (Fn 31) S 211.

113 Cicero Pro Cluentio 626481.

114 Idem 64f.

115 Idem 82.

116 Idem 66-81.

117 Stroh (Fn 31) S 211.

118 Idem S 312.

119 Cicero Pro Cluentio 1. 
will, ${ }^{120}$ und kündigt gleich am Anfang der Rede an, dass er vom Tatbestand nichts zu verheimlichen und auf jeden, vom Attius erwähnten Umstand zu reflektieren vorhat. ${ }^{121}$

Das Durchbrechen der Chronologie zeigt sich bei der Behandlung der gegen den Fall des Cluentius sprechenden praeiudicia und des Verbrechenskatalogs des Oppianicus am deutlichsten. Der Vorsitzende (iudex quaestionis) des Gerichtshofes im Oppianicusprozess, C Iunius wurde im Jahre 74 verurteilt und der Senat erliess noch im selben Jahr einen Beschluss, der es ermöglichte, dass die am iudicium Iunianum beteiligten Richter zur Verantwortung gezogen wurden. ${ }^{122}$ Im Jahre 73 wurde C Fidiculanius Falcula in zwei Prozessen freigesprochen, ${ }^{123} 72$ wurde P Septimius Scaevola wegen crimen repetundarum und zwischen 73 und 70 MAtilius Bulbus wegen crimen maiestatis verurteilt, im Jahre 70 erteilten die Zensoren M Aquilius, Ti Gutta, P Popilius und Cluentius eine Rüge, 69 wurden Popilius und Gutta wegen ambitus und Staienus wegen anderer Verbrechen verurteilt. ${ }^{124}$ Der Ankläger versuchte all diese Urteile von der Art der jeweiligen Anklage unabhängig als Konsequenz des iudicium Iunianum darzustellen. ${ }^{25}$ Cicero erstellt hiergegen eine künstliche, den Interessen der Verteidigung entsprechende Chronologie, die einerseits die Urteile als Folge der vom Volkstribun Quinctius geschürften invidia erscheinen lässt ${ }^{126}$ und andererseits mit eine Antiklimax die Aufmerksamkeit von den gewichtigeren Fällen ${ }^{127}$ (dh der litis aestimatio des Septimius Severus, ${ }^{128}$ den als unwichtig abgestempelten zensorischen Rügen, ${ }^{129}$ dem Testament des Egnatius ${ }^{130}$ und den Senatsbeschlüssen) ${ }^{131}$ auf seine eigene in den Verrinen formulierte Meinung lenkt. ${ }^{132}$ Hiermit erweckt er in seinen Hörern den Eindruck einer sich legenden invidia. ${ }^{133}$

Genauso deutlich wird die von Cicero der forensischen Taktik entsprechend erstellte Chronologie im Hinblick aufdie von Oppianicus dem Älteren begangenen und ihm zugeschriebenen Morde und Untaten. ${ }^{134}$ Der erste Mord: Oppianicus ermordete

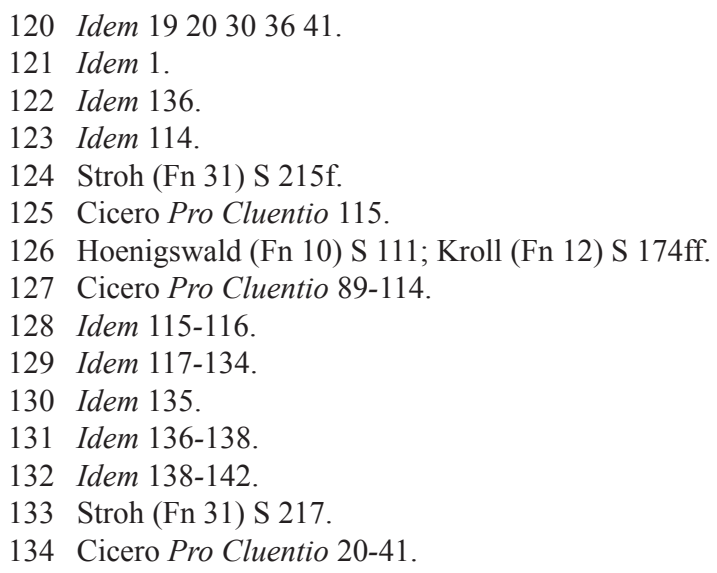


seine Ehefrau Cluentia, die Tante des Cluentius. ${ }^{135}$ Der zweite und dritte Mord: Oppianicus vergiftete die schwangere Ehefrau seines Bruders, des C Oppianicus und den eigenen Bruder, um an dessen Erbschaft zu kommen. ${ }^{136}$ Nach dem Tode seines Schwagers, des Cn Magius, der den jüngeren Oppianicus als Erben eingesetzt hatte, überredete Oppianicus der Ältere die schwangere Witwe zur Abtreibung und heiratete sie. ${ }^{137}$ Der vierte Mord und die Testamentsfälschung: Mit der Hilfe eines reisenden Giftmischers ermordete Oppianicus seine ehemalige Schwiegermutter Dianea, die ihn als Erben eingesetzt hatte, und liess das Testament, als er die Legate schon früher hatte tilgen lassen, neu schreiben und mit einem falschen Siegel versehen. ${ }^{138}$ Der fünfte Mord: Oppianicus liess M Aurius, den Sohn der Dianea, dem seine Mutter vierhunderttausend Sesterzen vermacht hatte, ermorden, von dem er erfuhr, dass er in Kriegsgefangenschaft geraten war und als Sklave in Gallien lebte. ${ }^{139}$ Der sechste, siebte und achte Mord: Oppianicus liess A Aurius, der ihm wegen der Ermordung des M Aurius mit einer Anklage gedroht hatte - und drei weitere Bürger aus Larinum - unter dem Vorwand der Proskriptionen töten. ${ }^{140}$ Der zehnte und elfte Mord: Oppianicus wollte Sassia, die Witwe des A Aurius heiraten, die aber nicht die Stiefmutter von dessen drei Söhnen werden wollte, und Oppianicus tötete deswegen zwei von seinen drei Söhnen und liess nur den jüngeren Oppianicus am Leben. ${ }^{141}$ Die Testamentsfälschung und der zwölfte Mord: Oppianicus liess, um sich als Erben einsetzen zu lassen, das Testament des Asuvius, eines Bürgers aus Larinum, fälschen und Asuvius ermorden; hiernach bestach er den triumvir capitalis Q Manlius, der eine Untersuchung wegen des Falles in die Wege leiten wollte. ${ }^{142}$

Cicero ändert diese Chronologie und gibt von den Verbrechen des Oppianicus in folgender Reihenfolge Rechnung: die Ermordung des $\mathrm{M}$ Aurius, ${ }^{143}$ des A Aurius und der drei Bürger aus Larinum, ${ }^{144}$ der zwei Söhne,,${ }^{145}$ der Cluentia, ${ }^{146} \mathrm{der}$ Schwägerin und des C Oppianicus, des Bruders, ${ }^{147}$ die Anstiftung zur Abtreibung, ${ }^{148}$ die Testamentsfälschung und die Ermordung des Asuvius, ${ }^{149}$ die Ermordung der

135 Idem 30.

136 Idem 30-32.

137 Idem 33-35.

138 Idem 40-41.

139 Idem 21-23.

140 Idem 23-25.

141 Idem 26-28.

142 Idem 36-39.

143 Idem 21-23.

144 Idem 23-25.

145 Idem 26-28.

146 Idem 30.

147 Idem 30-32.

148 Idem 33-35.

149 Idem 36-39. 
Dinaea und die Fälschung ihres Testaments. ${ }^{150}$ Es stellt sich die Frage, warum Cicero diesen Weg einzuschlagen „gezwungen“ ist. ${ }^{151} \mathrm{Da}$ die narratio in keiner direkten Verbindung mit dem Fall des Cluentius steht, kann der Redner auf die einzelnen Fälle nicht so eingehen, dass er diese mit Urkunden oder Zeugenaussagen zu beweisen versuchen könnte, sondern muss sich damit begnügen, den Anschein einer eingehenden Beweisführung zu erwecken. ${ }^{152}$ Eine eventuelle Beweisführung wäre durch die Unwahrscheinlichkeit des von Cicero vorgetragenen Kriminalromans zweifelsohne erschwert worden: Warum sollte der Serienmörder Oppianicus, dem seine eigenen Familienmitglieder zum Opfer fielen, der sich am Vermögen seiner Opfer bereicherte, der die Gattin seines Opfers heiratete, erst fünfzehn Jahre nach seinem ersten Mord zur Verantwortung gezogen worden sein; warum sollte er von mehreren als Erbe eingesetzt worden sein, obwohl es den Testierenden klar gewesen sein muss, dass sie damit ihren baldigen Tod herbeiriefen; warum sollte C Oppianicus den Mörder seiner Gattin zu seinem Erben gemacht haben; warum sollte Oppianicus nur zwei von seinen Söhnen ermordet und den dritten am Leben gelassen haben; warum sollte Oppianicus Aurius ermordet haben, obwohl er dessen Legat schon früher, als er das Testament der Dianea gefälscht hatte, aus dem Testament tilgte? ${ }^{153}$

Der Redner versucht die hier angeführten Gegenargumente erst gar nicht zu widerlegen, sondern er ist vielmehr bemüht, dass diese seiner Hörerschaft gar nicht in den Sinn kommen, dh er arbeitet anstatt mit offensichtlichen Lügen, mit subtilen Verschleierungen und raffiniertem Verschweigen bzw mit der willkürlichen Anordnung der Dramaturgie der Ereignisse. Dass sein Bestreben von Erfolg gekrönt wurde, wird auch dadurch bewiesen, dass auch die späteren Kommentatoren keinen Verdacht geschöpft haben und erst Winfried Stroh versucht hat, den tatsächlichen Hergang der Ereignisse zu rekonstruieren.

Dass Cicero die Ermordung des M Aurius an erster Stelle behandelt hatte, erwies sich als meisterhafter Kunstgriff, da er als „Beweis” für die um den Fall entstandenen Gerüchte und die von A Aurius ausgesprochenen Drohungen ${ }^{154}$ bzw für das Ausbleiben des Prozesses den Missbrauch der sullanischen Proskriptionen, dh die Ermordung des A Aurius mit Hilfe politischer Machenschaften vorbrachte. ${ }^{155}$ Mit dem politischen Einfluss des Oppianicus konnte der Redner zugleich erklären, warum der Serienmörder Oppianicus erst fünfzehn Jahre nach seinem ersten Mord zur Verantwortung gezogen worden ist. ${ }^{156}$ Die Fragen, die in Verbindung mit dem Tod der Dianea und ihrem Testament gestellt werden könnten, umgeht Cicero mit

150 Idem $40 \mathrm{f}$.

151 Stroh (Fn 31) S 220.

152 A Michel Rhétorique et philosophie chez Cicéron. Essai sur les fondaments philosophiques de l' art de persuader (Paris, 1960) S $257 \mathrm{ff}$.

153 Stroh (Fn 31) S 221.

154 Cicero Pro Cluentio 23.

155 Kroll (Fn 12) S 176.

156 Stroh (Fn 31) S 222. 
genauso grosser Genialität. Als er Dianea zum ersten Mal erwähnt, spricht er bloss von ihrer Krankheit und ihrem Tod bzw über die Existenz ihres Testaments, übergeht aber die Testamentsfälschung, ${ }^{157}$ und bringt erst später - nach einer kataraktartigen Aufzählung der Verbrechen des Oppianicus, was den neuen Mord gleichsam logisch macht - die Ermordung der Dianea und die Tatsache der Testamentsfälschung zur Sprache. ${ }^{158}$ Dass Oppianicus bereit war, seine eigenen Söhne zu ermorden, erklärt Cicero nicht aus dem Charakter des Oppianicus, sondern aus dem der Sassia, die zur Eheschliessung nur unter dieser Voraussetzung ihr Einverständnis gab - das von der den Mörder ihres Gatten heiratenden Sassia gezeichnete düstere Portrait ${ }^{159}$ schliesst den Mord nicht aus, sondern macht ihn gerade wahrscheinlich. ${ }^{160}$ Der Mangel an Beweisen hält Cicero davon nicht ab, aus der Not eine Tugend zu machen und die Richter daran zu erinnern, dass ihre Empörung im Vergleich zur Empörung jener Richter, die vor acht Jahren den Fall des Oppianicus untersucht und die Zeugenaussagen angehört hatten, als gering erscheinen wird. ${ }^{161}$

Die Ermordung der Cluentia (der ersten Gattin des Cluentius), der Schwägerin und des C Oppianicus (des Bruders) tut Cicero - angeblich um seine Rede nicht in die Länge zu ziehen - in aller Kürze ab. Allerdings veranlasst die Erwähnung der Cluentia nach Sassia - von der Cicero nicht behauptet, dass sie bis zu dessen Tod die Gattin des Oppianicus geblieben ist - die Hörerschaft mit gutem Grund zu jener Vermutung, dass Cluentia erst später, nach Sassia zur Gattin des Oppianicus wurde, und es kommt in ihnen der Verdacht nicht hoch, dass der Redner von Ereignissen vor dem Jahre 82 spricht. Es steht ausser Zweifel: Ciceros Ziel muss es gewesen sein, die Chronologie und damit auch die Hörerschaft gänzlich zu verwirren, da er die hier aufgezählten Morde nicht beweisen, höchstens beklagen konnte. ${ }^{162}$ Jene Geschenke, die Oppianicus Magia, der Witwe seines Schwagers, gegeben hat, lassen zwar nur auf Heiratsabsichten schliessen, aber Cicero stellt sie als merces abortionis dar, indem er sie mit der auf Anstiftung des Oppianicus vorgenommene Abtreibung von Magia in Verbindung bringt. ${ }^{163}$ Um die Ermordung der Dianea und die Fälschung ihres Testaments glaubhaft erscheinen zu lassen, ${ }^{164}$ fügt hier Cicero die Ermordung des Asuvius ein - in dessen Testament Oppianicus als Haupterbe eingesetzt worden ist -, was durch die Aussage des Avillius, eines Komplizen des Oppianicus unterstützt wird. Hierdurch wird das Beerben seiner Opfer zu einem der Hauptmotive der Untaten des Oppianicus, ${ }^{165}$ was die Ermordung der Dianea und die

157 Cicero Pro Cluentio 21f.

158 Idem $40 \mathrm{f}$.

159 Idem 12-16.

160 Stroh (Fn 31) S 222.

161 Cicero Pro Cluentio 29.

162 Stroh (Fn 31) S 223.

163 Cicero Pro Cluentio 34.

164 Idem 40f.

165 Idem 36-39. 
Fälschung ihres Testaments nur zu einer Steigerung der Motive des Asuvius-Falles macht. ${ }^{166}$

Die narratio der Cluentiana ist ein Paradebeispiel für den ordo artificialis - bzw für den mos Homericus ${ }^{167}$-, in der die von der utilitas causae bedingte Strategie die Stelle der als Tugend angerechneten perspicuitas übernimmt. Aufgrund dieser Strategie werden einerseits bei der Kette, andererseits bei der Darstellung der inneren Struktur der Ereignisse die glaubhafteren und besser belegbaren Elemente vor die schwer beweisbaren - oder gar unbeweisbaren - gestellt, damit gleichsam der Kredit und der Grund für die letzteren geschaffen werden. ${ }^{168}$

$4 \quad$ Um die Bravouren der Cluentiana technisch zusammenzufassen: Mit der getrennten Behandlung der Anklagepunkte wegen Bestechung und Giftmord verdoppelt Cicero sowohl die narratio, als auch die argumentatio; er fügt die normalerweise der narratio folgende propositio unmittelbar nach dem prooemium ein; bei der Behandlung sowohl des ersten, als auch des zweiten Anklagepunktes verschmelzen die argumentatio und die narratio ineinander; die als Abschluss eingefügte peroratio fliesst direkt aus der narratio; die extra causam eingefügten Erzählungen, die freie Behandlung der Chronologie bzw die gleichzeitige Verwendung des status collectionis und des status coniecturalis verstärken zusammen die Positionen des Verteidigers. Diese forensische Taktik versetzt den Leser bzw den Hörer gerade deswegen in Erstaunen, weil er kein einziges Mal das Gefühl bekommt, als wäre er das Opfer einer vorsätzlichen Irreführung seitens von Cicero, und was mehr ist: Die Teile der Erzählung reihen sich in einer scheinbar logischen Kette so aneinander, dass ausser Stroh fast alle Kommentatoren der Rede von der von Cicero aufgestellten Ordnung der Ereignisse ausgingen und versuchten, den historischen Tatbestand zu rekonstruieren. ${ }^{169}$

Cicero selbst beruft sich als beispielhaftes Exempel der Mischung der drei Stilarten auf seine Cluentiana, ${ }^{170}$ in der das in die Länge gezogene Exordium, die nüchtern kurzen Beschreibungen, die präzise Argumentation, die farbigen Erzählungen, die emotionalen Begründungen, der Pathos und die Ironie, der sprachliche Humor und die mit Leidenschaft eingeprägten Stichwörter, die zutreffenden Charakterisierungen, die auch Übertreibungen nicht entbehrenden Verallgemeinerungen, die mit Wucht gestellten Fragen und die invektivenartigen Exkurse in einer sonst nirgends gesehenen Harmonie verflochten werden. ${ }^{171}$ Cluentius wurde - dank Cicero freigesprochen, aber, wie wir es von Quintilian erfahren können, gestand der Redner

166 Stroh (Fn 31) S 224.

167 Quintilianus Institutio oratoria 7, 10, 11.

168 Stroh (Fn 31) S 224f.

169 Idem S 226f.

170 Cicero Orator 103. Vg1 Humbert (Fn 32) S 280.

171 Classen (Fn 26) S 105. 
auch zu, dass dies nur mit der geschickten Manipulation der Richter erreicht werden konnte. ${ }^{172}$ Vielleicht auch deswegen betrachtete Cicero seine Rede für Cluentius als eine der Höchstleistungen seiner rednerischen Laufbahn, ${ }^{173}$ wo ihm sowohl Quintilian, ${ }^{174}$ als auch Plinius der Jüngere, der diese oratio als die hervorragendste Gerichtsrede Ciceros lobpreiste, beipflichteten. ${ }^{175}$ Die Rede ist wahrlich beispielhaft gestaltet: Der Redner wechselt meisterhaft die Stilarten, bringt Pathos, einfache Beschreibungen und Humor miteinander in Einklang, stellt mit grosser Präzision abenteuerliche Ereignisse und dramatische Persönlichkeiten dar und verbindet die Zeitebenen und Argumente plastisch miteinander, ohne - ausser es steht in seinem Interesse - den Tatbestand noch rätselhafter zu machen. Er fesselte bis zum Schluss die Aufmerksamkeit seiner Hörerschaft und lenkte die Entscheidung der Richter in die von ihm gewünschte Richtung, da es ihm gelang - wie er es später selber gestand - ihnen Sand in die Augen zu streuen. ${ }^{176}$

\section{Abstract}

The speech for the defence in the criminal action (causa publica) of Aulus Cluentius Habitus, Cicero's longest surviving speech, dates back to the year 66 when Cicero was praetor. In certain respects, it is the jewel in Cicero's ars oratoria, since its narrative is vivid and full of twists and turns like a crime story. Events, scenes and time sequences follow one another in a dramatic, sometimes seemingly illogical fashion, but in view of the effect the orator seeks to attain, in an exactly premeditated sequence. One charge against Cluentius was that he had poisoned his stepfather, Statius Albius Oppianicus. Another charge was based on the criminal proceedings that had been instituted eight years previously, when Cluentius had charged Oppianicus with attempting to poison him, which resulted in Oppianicus being forced into exile. In the current lawsuit, however, the prosecution alleged that the court in the previous case had declared Oppianicus guilty purely because Cluentius had bribed the judges. The lex Cornelia de sicariis et veneficiis of 81 was applicable with regard to charges of poisoning. However, that law prohibited bribing only those judges who belonged to the order of senators, and Cluentius belonged to the order of knights. First, I outline the historical background of the oration, that is to say, the facts of the case; then, I turn my attention to the possibility of applying the lex Cornelia de sicariis et veneficiis to the case. Finally, I examine Cicero's oratorical strategy of addressing, modifying or distorting the charges and their chronology in order to back up his argument, which lawyers, too, will regard as brilliant.

172 Quintilianus Institutio oratoria 21721.

173 Cicero Orator 107f.

174 Quintilianus Institutio oratoria 41 35; 659.

175 Plinius epistulae 1204.

176 Quintilianus Institutio oratoria 21721. 